\title{
ACQUISITION, PROCESSING AND INVERSION OF MAGNETIC DATA IN ARCHAEOLOGICAL PROSPECTING
}

\author{
J. V. Herwanger ${ }^{\ddagger}$, H. R. Maurer, J. Leckebusch, A. G. Green \\ Institute of Geophysics, ETH-Hönggerberg, 8093 Zürich, Switzerland \\ ${ }^{\ddagger}$ now at: Department of Geology, Imperial College, Prince Consort Road, London SW7 2BP, UK \\ j.herwanger@ic.ac.uk
}

\section{INTRODUCTION}

Magnetic prospecting is a powerful tool for locating and characterizing archaeological features in the shallow subsurface. The introduction of inexpensive optically pumped magnetometers with a resolution of better than $0.1 \mathrm{nT}$ facilitates magnetic prospecting for small structures with low susceptibility contrasts. To take full advantage of this new tool, appropriate acquisition strategies and new data analysis techniques are required. For example, applications of various digital signal processing routines allow the locations of archaeological structures to be more precisely determined. Furthermore, 3-D inversions of magnetic data can provide reliable information on the depth extent and shape of the buried objects. These new technologies have been applied in a multidisciplinary study of an archaeological site in northern Switzerland.

\section{SURVEY AREA AND SUBSURFACE MODEL}

The Unterstammheim site contains remnants of an early medieval settlement that were "rediscovered" on air photographs in 1988. Subsequently, the area was chosen as a test site for applications of geophysical methods in archaeological prospecting. Principal targets of our investigation are pit-houses, an important form of accommodation during medieval times. Pithouses were holes in the ground that over time were filled with humus material and finegrained terrestrial sediments. Since the humus is expected to have a higher susceptibility than the unconsolidated host sediments (mostly gravels and sands), the pit-houses should be the source of low amplitude magnetic anomalies. It is assumed that the dominant components of these anomalies are due to induced magnetization.

\section{ACQUISITION}

An electric railway line very near the survey area produced high frequency $(16.67 \mathrm{~Hz})$ electromagnetic noise that had considerably higher amplitudes than the expected anomalies due to the archaeological targets. Acquiring magnetic data in a gradiometric configuration proved to be an effective means of significantly reducing the influence of this noise. Besides suppressing these undesired systematic effects, gradiometric data are expected to provide higher resolution of the very shallow structures (Blakely, 1995).

The pit-houses are expected to produce anomalies that are of the order of a few $\mathrm{nT} / \mathrm{m}$ or less. Successful delineation of such anomalies requires a high-precision measuring device. We have employed an optically pumped caesium magnetometer. By means of repeated measurements, we have shown that the magnetic gradient data can be reproduced to within an accuracy $\sim 0.05 \mathrm{nT} / \mathrm{m}$.

Reliable identification of small-scale features (2-5m in extent) requires not only an accurate recording system but also dense spatial sampling of the investigation area. Extensive tests with different grid spacings has demonstrated that data sampling of $0.25 \mathrm{~m}$ or less is necessary to prevent spatial aliasing and to reliably image the expected small targets. 
Our magnetic gradient data set was collected over a $60 \times 220 \mathrm{~m}$ area by a two-person crew during a 6-day period. It comprises $251^{\prime} 000$ data points. A small portion of the total data set is shown in Figure 1.

\section{PROCESSING}

To extract maximum information from the magnetic gradient data, the following digital signal processing techniques were successfully applied:

- gentle smoothing in the space domain using a 9-point Hanning filter to remove any residual effects of the railway line;

- low-cut Butterworth filtering in the wavenumber domain (cutoff wavelength of $10 \mathrm{~m}$ ) to separate anomalies of archaeological origin from longer wavelength regional fields;

- reduction-to-the-pole, which under the assumption of dominant induced magnetization and symmetric sources, centers the anomalies over the causative bodies (Blakely, 1995); in our examples, the centers of the anomalies move northwards by as much as $0.5 \mathrm{~m}$ (Fig. 1);

- vertical derivative of the magnetic gradient data (effectively the $2^{\text {nd }}$ vertical derivative of the total magnetic field) to better define the lateral boundaries of buried objects.

\section{INVERSION}

In order to estimate the depth extent and shape of the pit-houses, a new 3-D magnetic inversion technique has been developed and applied. An important constraint on the inversions is offered by the observation that, except for the pit-houses, the surface humus layer has a relatively constant thickness over the survey area. Sources of the pit-house anomalies are therefore assumed to lie at a constant depth. The subsurface model is represented by a number of vertical prisms with upper surface depths set to the regional thickness of the humus layer (Fig. 2). The principal unknown parameters are the lengths of the individual prisms. The susceptibility contrast $\kappa$ between the humus and the host gravels and sands is assumed to be constant and is either (i) measured in the laboratory from field samples, (ii) measured in the field using in situ susceptibility meters, or (iii) inferred from the results of a suite of inversions. Expected smoothness of the subsurface structures provides a further constraint on the inversion process.

Before inverting the recorded data, extensive tests with synthetic data were performed. A major objective was to study the properties and resolution power of the inversion algorithm. An example of these tests is presented in Figure 3. It shows cross-sections through a variety of hypothetical pit-houses. These tests demonstrate the feasibility of reliably reconstructing the shapes of the pit-houses. The inversion algorithm works particularly well for low-angle sidewalls (Figs. 3a to 3c), but tends to oscillate for steeper dipping features (Fig. 3d).

Inversion results for the subset of recorded data shown in Figure 1 are depicted in Figure 4. Several depressions are observed in the subsurface model. The largest depression probably represents a medieval pit-house. Note the steepness of its side walls.

\section{CONCLUSIONS}

Magnetic prospecting is capable of yielding detailed "images" of buried archaeological features when appropriate acquisition, processing and inversion strategies are employed. To obtain meaningful results, acquisition parameters must be chosen carefully. Digital signal processing can improve significantly the visibility and location accuracy of subsurface bodies, whereas detailed information on pit-house shapes and depths can be obtained via 3-D inversions of the magnetic gradiometric data.

\section{REFERENCES}

Blakely, R.J., 1995, Potential Theory in Gravity and Magnetic Applications, Cambridge University Press, Cambridge, 441pp. 

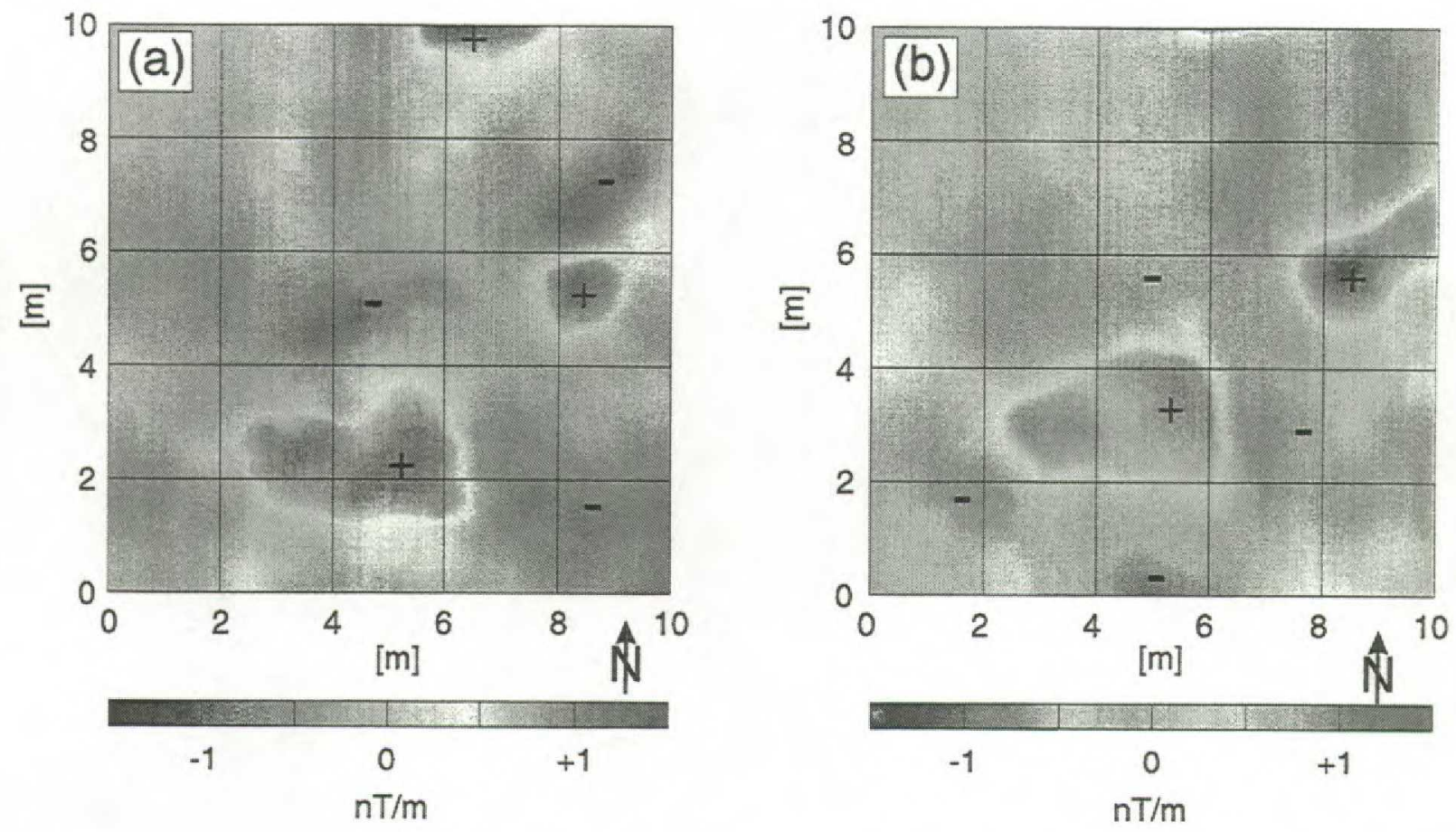

Figure 1: (a) Unprocessed magnetic gradient data and (b) the same data after being reduced-to-the-pole. Note the northward shift of the large anomaly by $\sim 0.5 \mathrm{~m}$ as result of the filtering; the shape of the anomaly becomes approximately symmetric after filtering, suggesting it may be the result of induced magnetization.

\section{a) subsurface model}

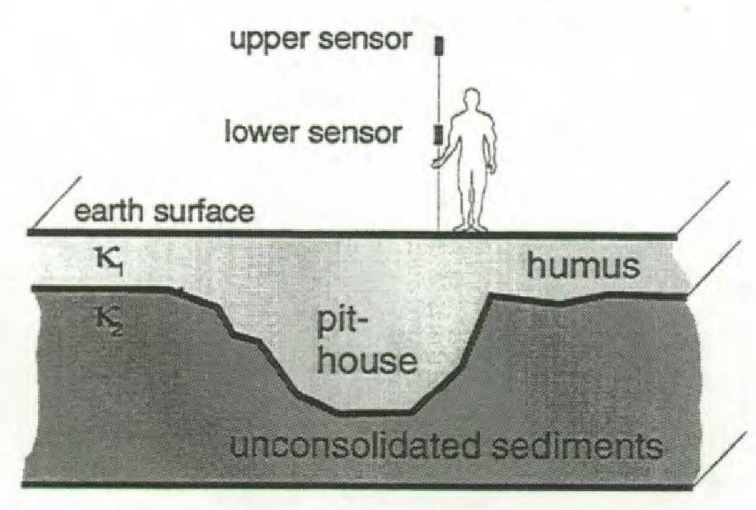

b) model used for 3D-Inversion

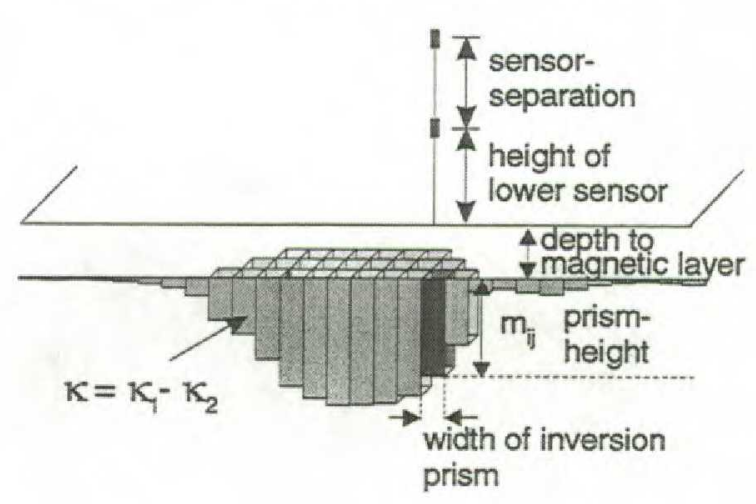

Figure 2: (a) Sketch of subsurface model expected at Unterstammheim. b) equivalent model to (a) used for the 3-D inversion. 
a) angle of pit-wall: $30 \mathrm{deg}$.

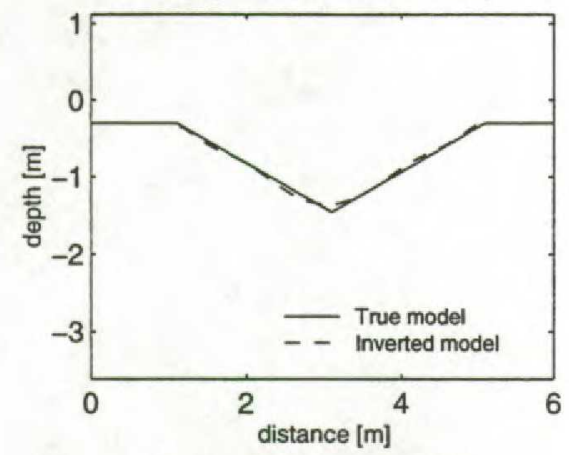

c) angle of pit-wall: $60 \mathrm{deg}$.

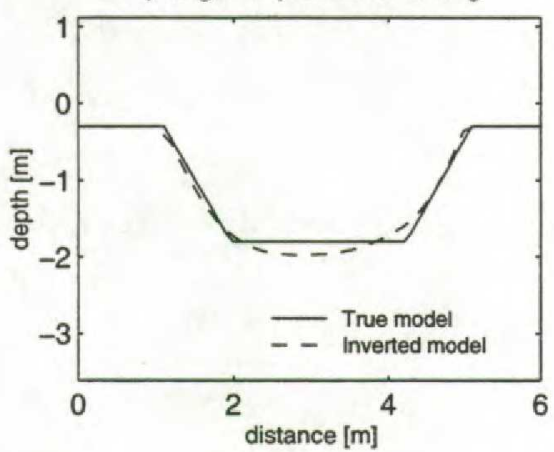

b) angle of pit-wall: 45 deg.

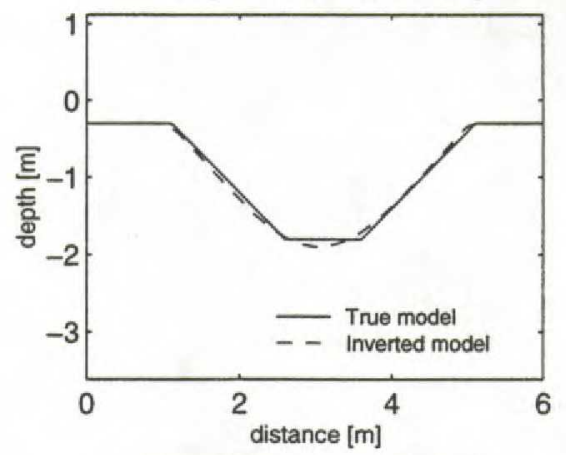

d) angle of pit-wall: 75 deg.

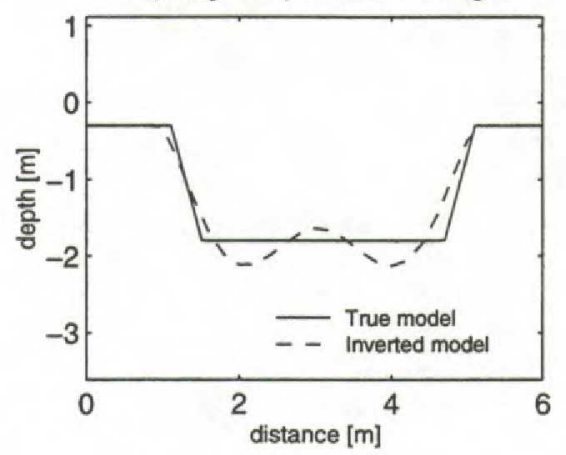

Figure 3: Test of the inversion algorithm on synthetic data. Shown are depth sections through pit-houses. Solid lines represent the synthetic (true) subsurface model, and the dashed lines represent the reconstructed (inversion) model. The slope angle of the pit-house wall is successively increased from $30^{\circ}$ to $75^{\circ}$.

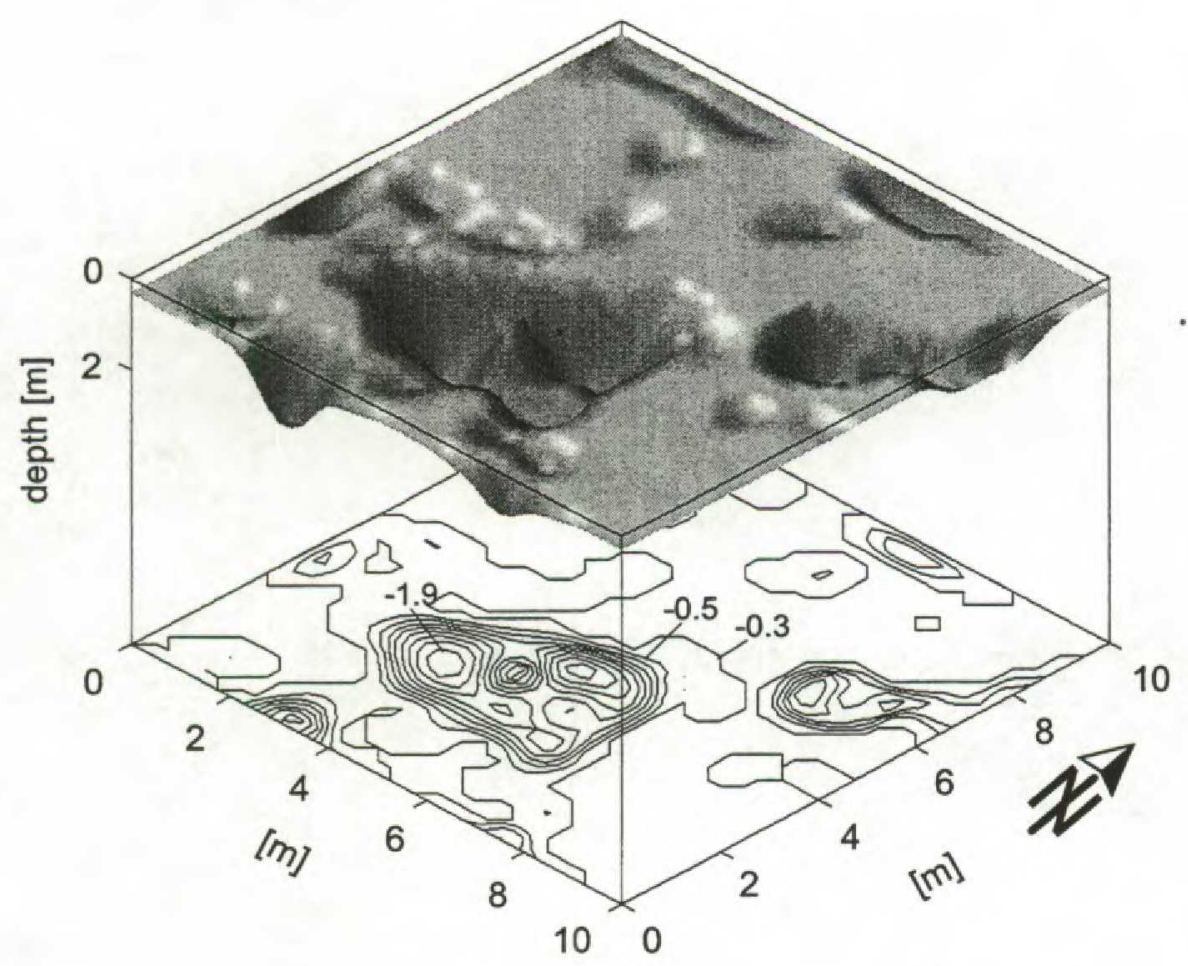

Figure 4: Subsurface model for the data set shown in Figure 1a. Pictured is the boundary between the humus and the unconsolidated sediments (Fig. 2). The upper surface shows in shaded relief format and the lower displays depth contour lines. Contour interval is $0.2 \mathrm{~m}$. 